\title{
Estimation of Signal Arrival Times in the presence of Delay Noise
}

\author{
Sarvesh Bhardwaj \\ ECE Department \\ University of Arizona \\ sarvesh@ece.arizona.edu
}

\author{
Sarma B.K.Vrudhula \\ ECE Department \\ University of Arizona \\ sarma@ece.arizona.edu
}

\author{
David Blaauw \\ EECS Department \\ University of Michigan \\ blaauw@umich.edu
}

\begin{abstract}
Delay due to capacitive coupling of interconnects has become an important reliability issue in the design of nanometer circuits. In this paper we present a probabilistic approach towards analyzing the impact of capacitive coupling noise on signal delay. The variation in the delay is due to the variation in the relative arrival times of the aggressors and the victim. We derive expressions for the moments of the victim voltage in the presence of noise. From these we compute estimates of the earliest and latest possible arrival times of the victim. We compare the analytical results with Monte Carlo simulations using SPICE. Even though the analytical calculations are 200 times faster than the Monte Carlo simulations, the differences in the estimates of the mean and standard deviation of the arrival time is no more than $2.8 \%$. In addition, the width of the timing intervals using the proposed approach is reduced by as much as $48 \%$ with a confidence level of 0.984 . That is $98.4 \%$ of the Monte Carlo simulations result in an arrival time that falls within the derived interval which is $48 \%$ shorter.
\end{abstract}

\section{Introduction}

The reduction in spacing between interconnects in nanometer scale circuits has caused the cross coupling capacitance to become the dominant component of the total interconnect capacitance [20, 22]. Similar trends are also seen with inductive coupling primarily due to higher clock frequencies [5, 11]. The term delay noise is used to describe the situation when switching on a net (aggressor) results in a change in the timing characteristics of the neighbouring net (victim). Delay noise can result in the victim getting delayed and thus resulting in a timing violation. The timing violation can be a hold time or setup time violation depending on the relative switching directions of the victim and aggressor.

Interconnect coupling noise has become a major concern in the design of nanometer scale circuits. As a result, noise simulators [13, 20] have become an indispensable tool in their design. Detailed simulation of distributed RC models of an industrial circuit using noise simulators is computationally prohibitive as they often have hundreds of thousands of nets. Consequently, accuracy is sacrificed for speed by using linear models of the gates that drive the victim and aggressor nets [13, 21], employing reduced order models [14, 15 ] in the solution of the network equations, and using superposition to process multiple aggressors.

A key issue in noise simulation is the alignment of aggressor waveforms $[3,6,13,20,21]$. Associated with each net is an interval specifying the earliest (EAT) and latest arrival time (LAT) of that signal. The variations in gate and interconnect delays as well as the variation in lengths of different paths all contribute to the uncertainty of the arrival time within this interval. Noise simulators assume the worst case scenario by fixing the arrival times of the ag- gressors so that the peaks of the noise waveforms are aligned [3, 13, $20,21]$. The worst case alignment results in the maximum delay in the arrival time of the victim [6]. Even though the likelihood of realizing a worst-case composite noise is relatively small, this pessimistic approach can result in a significant reduction in the clock frequency.

Much work has been done toward analyzing signal delay in the presence of coupling. The main objective is to determine the new timing interval (earliest and latest signal arrival times) in the presence of coupling noise for use in static timing analysis (STA). The problem is complicated because the coupling capacitance is not constant as the victim and aggressor switch $[8,17]$, and because of the interdependence of the aggressor and victim arrival times [17]. An iterative approach is needed to compute the timing interval. This is done by starting with an initial window and then expanding or contracting it until the solution converges [2, 4, 24]. The windows thus obtained can be used for STA [18].

Logic and timing correlations $[7,12]$ can also be used to reduce the pessimism in noise analysis. The set of aggressors for a given victim can be reduced based on functional analysis of the signals [10]. However the computational complexity of determining logic and timing correlations is very high, and of limited benefit since physical proximity of signals and functional dependence among them might be unrelated.

Even after pruning the nets, the worst case assumptions incorporated into noise simulators often result in a very large number of timing violations being reported. One way to reduce this pessimism is to perform a sufficiently large number of Monte Carlo simulations, varying the arrival times of the victim and aggressor signals over their respective timing windows. Such an approach will yield a fairly accurate estimates of the arrival times in the presence of noise, but requires an enormous amount of computation time. What is needed is an analytical method that will yield estimates of the arrival time of a signal in the presence of noise, while taking into account the variations in the arrival times of the aggressor signals. This requires modeling the victim waveform as well as the noise waveform due to each aggressor to construct a composite victim waveform. Then the delay of the victim as a function of relative signal arrival times of the victim and aggressors can be computed [19]. In [8] the delay uncertainty of a victim net resulting from varying the slew rate of a single aggressor and a victim are studied.

In this work we present an approach that reduces the pessimism in obtaining the earliest arrival time (EAT) and latest arrival time (LAT) of a signal in presence of noise by taking into account the variability of the arrival times in absence of noise. The proposed method will help designers to accurately predict the timing window of a signal without having to perform time consuming Monte 


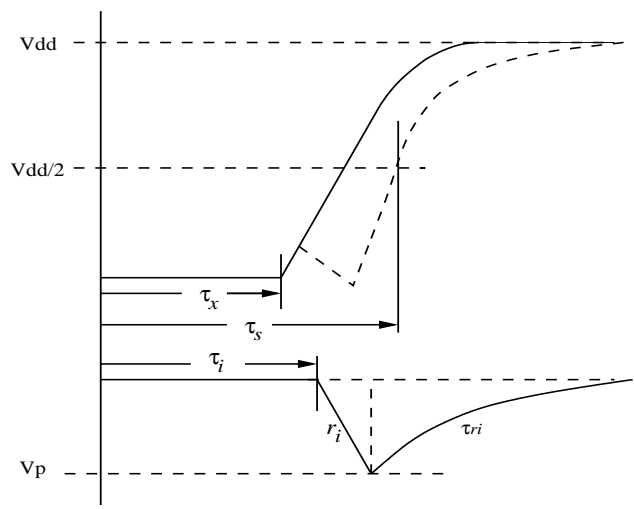

Figure 1: Victim and Noise waveforms.

Carlo simulations. Similar work has been done in [23] for functional noise. Our method permits making a tradeoff between the uncertainty in the estimate and the length of the timing window. We compare our results with Monte Carlo simulations on nets extracted from a high performance industrial processor. The results show that the approach leads to as much as $50 \%$ reduction in the width of the timing window with only a $3 \%$ decrease in the probability associated with the timing window.

The organization of rest of the paper is as follows: Section 2 contains a description of the noise and the victim models. It also shows a way to obtain the EAT and LAT using the moments of the victim in presence of noise. A discussion of the assumptions is given in Section 3. The expressions for the $k$ th moment of the victim and aggressor waveforms are derived in Section 4. Finally, in Section 5 we present a comparison of the theoretical results and Monte Carlo simulations using nets extracted from an industrial processor circuit.

\section{The Victim and Noise Models}

A victim with a set of $n$ aggressors is referred to as a cluster of size $n$. Since we are examining delay noise, we assume that the victim is switching in from logic 0 to logic 1 and the aggressor is switching from logic 1 to logic 0 .

The victim and the aggressor nets are modeled as a RC ladder network. Since all the elements in our circuit are linear, we can use the principle of superposition to model the voltage at a given net by adding the voltages of the victim in absence of noise, denoted by $X(t)$, and the noise waveform resulting from the ith aggressor is denoted by $Z_{i}(t)$. The general form of the noise waveform seen on the victim due to aggressor switching is a sum of weighted decaying exponentials. We model the victim and the noise waveforms as given in [19].

The victim is assumed to be a rising exponential with a rise time of $\tau_{r}$, maximum voltage $V_{d d}$ and switching time $\tau_{x}$. Figure (1) shows the victim and the noise waveforms. The switching times, $\tau_{i}$ and $\tau_{x}$, are considered to be random variables with triangular distributions over $\left[\eta_{i}-a_{i}, \eta_{i}+a_{i}\right]$ and $\left[\eta_{x}-a_{x}, \eta_{x}+a_{x}\right]$ respectively. Here $\eta_{i}-a_{i}$ and $\eta_{i}+a_{i}$ represent the Earliest Switching Time, EST and the Latest Switching Time, LST respectively. Also $\eta_{i}$ and $\eta_{x}$ denote the mean of the switching times. The length of the switching time windows is given by $2 a_{i}$ and $2 a_{x}$. The corresponding density function of switching time for the ith aggressor is

$$
f_{\tau_{i}}(\tau)=\left\{\begin{array}{lr}
\frac{1}{a_{i}^{2}}\left(a_{i}-\left|\tau-\eta_{i}\right|\right)^{2} & \eta_{i}-a_{i} \leq \tau<\eta_{i}+a_{i} \\
0 & \text { otherwise }
\end{array}\right.
$$

Since $\tau_{i}$ is a random variable, $Z_{i}(t)$ is a random variable for a fixed value of $t$. Let $S_{n}(t)$ represent the resulting waveform on a given victim net in a cluster of size $n$. As stated earlier, using the principle of superposition, we can represent $S_{n}(t)$ as the sum of the victim in absence of noise and the noise due to each aggressor. We assume that the random variables $Z_{i}(t), i=1,2, \ldots, n$, are independent. Thus we have

$$
S_{n}(t)=X(t)+Z_{1}(t)+Z_{2}(t)+\cdots+Z_{n}(t) .
$$

The arrival time of a signal is defined as the time after which the signal value is more than the threshold voltage. In the presence of noise the resulting victim waveform can cross the threshold voltage multiple times. In such a case we are interested in finding the last crossing of the waveform. $S_{n}(t)$ being a continuous and nonstationary process, obtaining the statistics of the arrival time for this process is not possible analytically. The next best thing we can do is to accurately estimate the arrival time window within some confidence limit. This is done by first deriving the expression for the $k t h$ moment of the victim in presence of coupling noise. Let $\mu_{v(t)}$ and $\sigma_{v(t)}$ represent the mean and the standard deviation of the victim waveform in presence of noise. That is, $\mu_{v(t)}=\mathbf{E}\left[S_{n}(t)\right]$ and $\sigma_{v(t)}=\sqrt{\mathbf{E}\left[\left(S_{n}(t)-\mu_{v(t)}\right)^{2}\right]}$ (see Equation 4). Similarly let $\mu_{t}$ and $\sigma_{t}$ represent the mean and the standard deviation of the victim arrival times. The time at which the mean of the victim waveform crosses the threshold voltage is an estimate of the mean of the arrival times $\left(\mu_{t}\right)$ of the victim in presence of noise. To find the timing window we define the two voltage waveforms as follows:

$$
\begin{aligned}
V_{k}^{-}(t) & =\mu_{v(t)}-k \sigma_{v(t)} \\
V_{k}^{+}(t) & =\mu_{v(t)}+k \sigma_{v(t)}
\end{aligned}
$$

Here $k$ is defined as the Reliability Factor. Increasing the value of $k$ increases the size of the timing window as well as the reliability of the circuit. Thus we define the arrival time window as follows

DEFINITION 2.1. Let $t_{k}^{-}$denote the latest time t such that $V_{k}^{-}(t)=$ $V_{d d} / 2$. Similarly, let $t_{k}^{+}$denote the latest time $t$ such that $V_{k}^{+}(t)=$ $V_{d d} / 2$. Then, $t_{k}^{+}$and $t_{k}^{-}$denote the upper and the lower limit of the arrival time window respectively.

Both $t_{k}^{+}$and $t_{k}^{-}$are obtained by solving $\mu_{v(t)}+k \sigma_{v(t)}=V_{d d} / 2$ and $\mu_{v(t)}-k \sigma_{v(t)}=V_{d d} / 2$, for $t$, respectively. We compute the voltage waveforms for different values of $k$, and find the corresponding arrival times. These arrival times form the EAT and the LAT of the victim in presence of noise. Note that the intervals [EAT, LAT] have a probability assigned to them. Therefore, it is indeed possible that a realization of the circuit may result in the arrival time falling outside this interval. Clearly, in statistical based design, we must determine such an interval at a prescribed level of confidence. We outline the entire procedure of computing the final arrival times window for a signal in Figure (2).

\section{Discussion of Assumptions}

Our approach is based on the following assumptions:

1. the switching times of the aggressors and the victim have a triangular distribution,

2. the noise waveform during the initial part (rising or falling) is linear, and

3. the individual aggressor and the victim signals are independent 


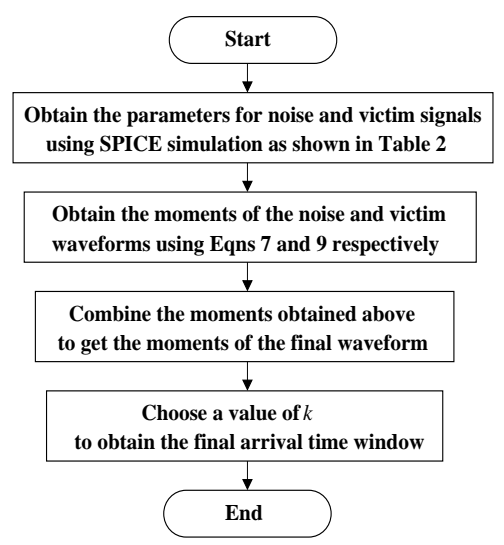

Figure 2: Flowchart for computing the Timing Windows

A triangular distribution for the signal switching time is reasonable since it is finite and unimodal. The alternative of a uniform distribution is unrealistic. The results presented in this paper are easily modified to accomodate non-symmetric triangular distributions. The second assumption is also not too severe a restriction since the inital part of the noise waveform due to each aggressor follows the steep falling or rising transition of the aggressor. It was included to simplify the algebra. It has no significant impact on the accuracy. The assumption that the aggressors are independent is an orthogonal issue. Under a zero delay model, determination of logic correlations is done separately. This would identify the set of "real" aggressors for a given victim. The approach presented in this paper would still be valid. Temporal correlations are far more difficult to model. At present we cannot account for such dependencies.

\section{Moments of the Coupled Victim}

In this section we derive the moments of the victim in a cluster of size $n$. Since $S_{n}(t)$ can be written as shown in (2), the moments of the victim in presence of noise can be written as

$$
\mathbf{E}\left[S_{n}^{k}(t)\right]=\mathbf{E}\left[\left(X(t)+Z_{1}(t)+Z_{2}(t)+\cdots+Z_{n}(t)\right)^{k}\right]
$$

Hence to obtain the moments of the coupled victim, we first need to derive the moments of the noise and the victim. For this, we first derive the moments of the individual noise waveforms. The $k t h$ moment of the individual noise waveform is given by [1]

$$
\begin{aligned}
\mathbf{E}\left[Z_{i}^{k}(t)\right]= & \frac{(-1)^{k}\left(r_{i}\right)^{k}}{a_{i}^{2}(k+1)(k+2)}\left[\left(t-\alpha_{1}\right)^{k+2}+\right. \\
& \left(t-\gamma_{1}\right)^{k+2}-2\left(t-\beta_{1}\right)^{k+2}- \\
& (k+2)\left\{2\left(\beta_{1}-\eta_{i}\right)\left(t-\beta_{1}\right)^{k+1}-\right. \\
& \left(\alpha_{1}-\eta_{i}+a_{i}\right)\left(t-\alpha_{1}\right)^{k+1}+ \\
& \left.\left.\left(\eta_{i}+a_{i}-\gamma_{1}\right)\left(t-\gamma_{1}\right)^{k+1}\right\}\right]+ \\
& \left(-V_{p}\right)^{k}\left(\frac{\tau_{r i}}{a_{i}^{2} k}\left(t-\eta_{i}+a_{i}-V_{p} / r i\right)-\right. \\
& \left.\frac{\tau_{r i}^{2}}{a_{i}^{2} k^{2}}\left(1-e^{-k\left(t-\eta_{i}+a_{i}-V_{p} / r i\right) / \tau_{r i}}\right)\right)
\end{aligned}
$$

the above expression is for $t \in\left[\eta_{i}-a_{i}+V_{p} / r_{i}, \eta_{i}+V_{p} / r_{i}\right]$ similar expressions can be derived for the rest of the cases as well. the values of $\alpha_{1}, \beta_{1}$ and $\gamma_{1}$ for the condition $V_{p} / r_{i} \geq a_{i}$ are given in
Table 2: Parameters of the victim and noise waveforms

\begin{tabular}{|c|c|c|c|}
\hline Signal & Peak Volt. (V) & Rise time (ns) & Fall time (ns) \\
\hline Victim & 1.35 & 26.20 & NA \\
\hline Agg. 1 & -0.408 & 26.178 & 0.962 \\
\hline Agg. 2 & -0.515 & 26.313 & 2.065 \\
\hline Agg. 3 & -0.040 & 26.193 & 0.775 \\
\hline
\end{tabular}

Table 1 where

$$
\begin{array}{ll}
t_{1}=\eta_{i}-a_{i} & t_{4}=\eta_{i}+V_{p} / r_{i} \\
t_{2}=\eta_{i}-a_{i}+V_{p} / r_{i} & t_{5}=\eta_{i}+a_{i} \\
t_{3}=\eta_{i} & t_{6}=\eta_{i}+a_{i}+V_{p} / r_{i}
\end{array}
$$

The moments of the total noise can be computed from the individual noise moments using the binomial expansion recursively. The product terms in this expansion are simply the products of the previously computed moments of individual noise waveforms. We now derive the $k$ th moment of the Victim. Here we give the $k$ th moment for $t \in\left(\eta_{x}-a_{x}, \eta_{x}\right)$, similar expressions can be obtained for rest of the cases.

$$
\begin{aligned}
\mathbf{E}\left[X^{k}(t)\right]= & 1+\frac{V_{d d}^{k}\left(t-\eta_{x}+a_{x}\right)}{a_{x}^{2}} \sum_{j=1}^{k}\left(\begin{array}{l}
k \\
j
\end{array}\right)(-1)^{j} \frac{\tau_{r}}{j}- \\
& \frac{V_{d d}{ }^{k}}{a_{x}^{2}} \sum_{j=1}^{k}\left(\begin{array}{c}
k \\
j
\end{array}\right)(-1)^{j} \frac{\tau_{r}^{2}}{j^{2}}\left(1-e^{-j\left(t-\eta_{x}+a_{x}\right) / \tau_{r}}\right)
\end{aligned}
$$

A closed form for the above summation was not possible. So we numerically compute the moments of the victim. The $k$ th moments of the noise and the victim can be combined to obtain the $k$ th moment of the victim in presence of noise.

\section{Experimental Results}

We used an RC network model of a cluster extracted from a high performance industrial processor. SPICE was used for performing the Monte Carlo simulations by varying the switching times of the victim and the aggressors. The parameters for the noise and the victim waveforms were obtained after one simulation for each net. The model parameters are shown in Table 2. The parameters of the probability distribution were taken to be different for all the nets. The logic threshold voltage (voltage above which the signal is assumed to be at logic 1$)$ was taken to be $V_{d d} / 2(0.675 \mathrm{~V})$. The cluster size (number of aggressors) was 3 . The figures (3) and (4) show both the calculated and simulated mean and the variance of the victim voltage respectively in the presence of noise. We see that the error in the region of interest (close to the Threshold voltage) is less than $1 \%$ for the mean.

The distribution function of the simulated arrival times of the victim in presence of noise and in absence of noise were constructed. Figure (5) shows the two distribution functions. The distribution of the victim in absence of noise is shifted to the right by $11.18 \mathrm{~ns}$ for better visibility. From these curves, we see that the mean delay on the victim is $11.12 n$ s (or close to $30 \%$ ). We also see that the shape of the distribution function of the victim in presence of noise is considerably different from the one in absence of noise. This is because the effect of noise on the victim is equivalent to convolving the density functions of the victim and the aggressors which will result in a similar waveform.

Four different test cases were generated by varying the switching windows of the nets. The widths of the switching windows varies considerably over all the cases. Figure 6 shows the $\mu_{v(t)}+1.5 \sigma_{v(t)}$ and $\mu_{v(t)}-1.5 \sigma_{v(t)}$. This figure shows the calculation of the EAT 
Table 1: Values of $\alpha_{1}, \beta_{1}$ and $\gamma_{1}$

\begin{tabular}{|c|c|c|c|c|c|c|c|}
\hline Range t & $\left(-\infty, t_{1}\right]$ & $\left(t_{1}, t_{2}\right]$ & $\left(t_{2}, t_{3}\right]$ & $\left(t_{3}, t_{4}\right]$ & $\left(t_{4}, t_{5}\right]$ & $\left(t_{5}, t_{6}\right]$ & $\left(t_{6}, \infty\right)$ \\
\hline$\alpha_{1}$ & $t$ & $t_{1}$ & $t-V_{p} / r_{i}$ & $t-V_{p} / r_{i}$ & $t-V_{p} / r_{i}$ & $t-V_{p} / r_{i}$ & $t-V_{p} / r_{i}$ \\
\hline$\beta_{1}$ & $t$ & $t$ & $t$ & $\eta_{i}$ & $t-V_{p} / r_{i}$ & $t-V_{p} / r_{i}$ & $t-V_{p} / r_{i}$ \\
\hline$\gamma_{1}$ & $t$ & $t$ & $t$ & $t$ & $t$ & $\eta_{i}+a_{i}$ & $t-V_{p} / r_{i}$ \\
\hline
\end{tabular}

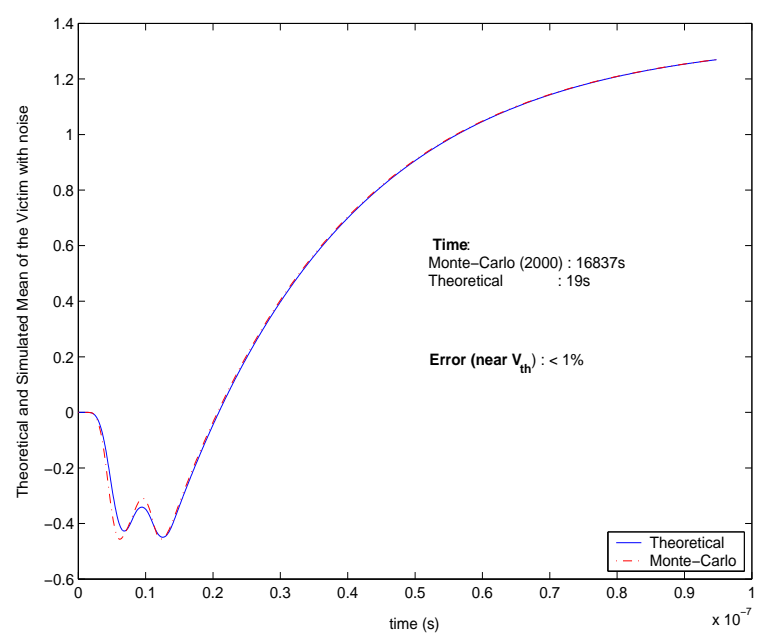

Figure 3: Means of the Victim waveform.

Table 3: Comparison of the Theo. and Sim. Arrival Times

\begin{tabular}{|c|c|c|c|c|}
\hline \multirow{2}{*}{ Case } & Sim. & \multicolumn{3}{|c|}{ Theoretical } \\
\cline { 2 - 5 } & EAT/LAT & EAT/LAT & Prob. & Win. size red. \\
\hline I & $36.92 / 40.47$ & $38.18 / 40.02$ & 0.962 & $48.2 \%$ \\
\hline II & $37.3 / 41.66$ & $38.26 / 40.94$ & 0.956 & $38.6 \%$ \\
\hline III & $48.21 / 58.65$ & $50.02 / 56.45$ & 0.97 & $38.5 \%$ \\
\hline IV & $45.12 / 56.08$ & $47.07 / 52.67$ & 0.984 & $48.91 \%$ \\
\hline
\end{tabular}

and the LAT of the victim waveform in presence of noise. The EAT and the latest arrival times for the rest of the cases is listed in Table (3). This table also shows the percentage of the simulated arrival times that fall in this window. The percentage reduction in the size of the window is also shown. We see that even after reducing the size of the window by as much as $48 \%$, the probability of the arrival times falling within this window reduces by only $1.6 \%$. That is $98.4 \%$ of the total arrival times fall within this window. This result also reflects on the amount of pessimism associated with the worst case analysis and how to reduce it.

Figure( 7) shows the percentage of signal arrival times falling in the timing window with respect to $k$. We see from the figure that as $k$ is increased, the percentage of events that fall in the arrival time window increases. For $k$ greater than 1.5, the percentage for all the cases is greater than $95.6 \%$. Thus choosing a $k$ greater than 1.5 gives a very good estimate of the arrival time window without sacrificing the accuracy.

Since the convolution of a large number of triangular distributions tends to Gaussian, we estimate the emperically obtained distribution with a Gaussian and compare the mean and standard deviation of this with the theoretically obtained mean and standard deviation. Table (4) shows the theoretical values and as well as those obtained by Monte Carlo simulation. We see that the values match within a maximum error of $2.8 \%$. Thus we can use these values to give accurate confidence limit on the arrival times.

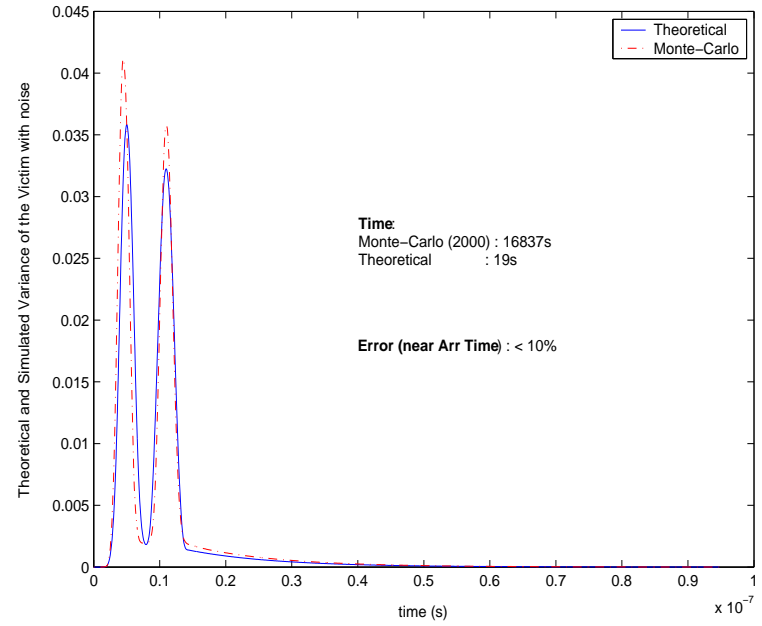

Figure 4: Second Central Moments of the Victim waveform.

Table 4: Simulated vs. Theoretical Mean and Std. dev.

\begin{tabular}{|c|c|c|c|c|c|c|}
\hline \multirow{2}{*}{ Case } & \multicolumn{2}{|c|}{ Simulated } & \multicolumn{2}{c|}{ Theoretical } & \multicolumn{2}{c|}{ Error } \\
\cline { 2 - 7 } & $\mu(\mathrm{ns})$ & $\sigma(\mathrm{ns})$ & $\mu(\mathrm{ns})$ & $\sigma(\mathrm{ns})$ & $\mu(\mathrm{ns})$ & $\sigma(\mathrm{ns})$ \\
\hline I & 38.7 & 0.606 & 39.1 & 0.614 & $1 \%$ & $1.3 \%$ \\
\hline II & 39.41 & 0.896 & 39.62 & 0.887 & $2 \%$ & $1 \%$ \\
\hline III & 53.15 & 2.09 & 53.46 & 2.14 & $0.6 \%$ & $2.8 \%$ \\
\hline IV & 49.83 & 1.83 & 50.00 & 1.87 & $2 \%$ & $2.2 \%$ \\
\hline
\end{tabular}

\section{Conclusions}

In this paper we presented a new approach to analyze delay noise. The objective was to get an estimate of the arrival times of a victim in presence of cross-coupling of adjacent nets without performing the time consuming Monte Carlo simulations. The theory was applied on a cluster extracted from a high performance microprocessor and the results were verified by performing Monte Carlo simulations using SPICE. The results show a very good agreement between the theoretical and simulated values with the maximum error being $2.8 \%$. The approach reduces the pessimism inherent in the worst case analysis by as much as $48 \%$.

\section{REFERENCES}

[1] http://www.ece.arizona.edu/ sarvesh/pubs.

[2] R. Arunachalam, K. Rajagopal, and L. T. Pileggi. TACO: timing analysis with coupling. In Proc. DAC, pages 266-269, 2000.

[3] L. H. Chen and M. Marek-Sadowska. Aggressor alignment for the worst-case coupling noise. IEEE Trans. on CAD, 20(5):612-621, May 2001.

[4] P. Chen and K. Keutzer. Towards true crosstalk noise analysis. In Proc. ICCAD, pages 132-137, 1999.

[5] K. Gala, V. Zolotov, R. Panda, B. Young, J. Wang, and D. Blaauw. Taco: timing analysis with coupling. In Proc. $D A C$, pages $63-68,2000$. 


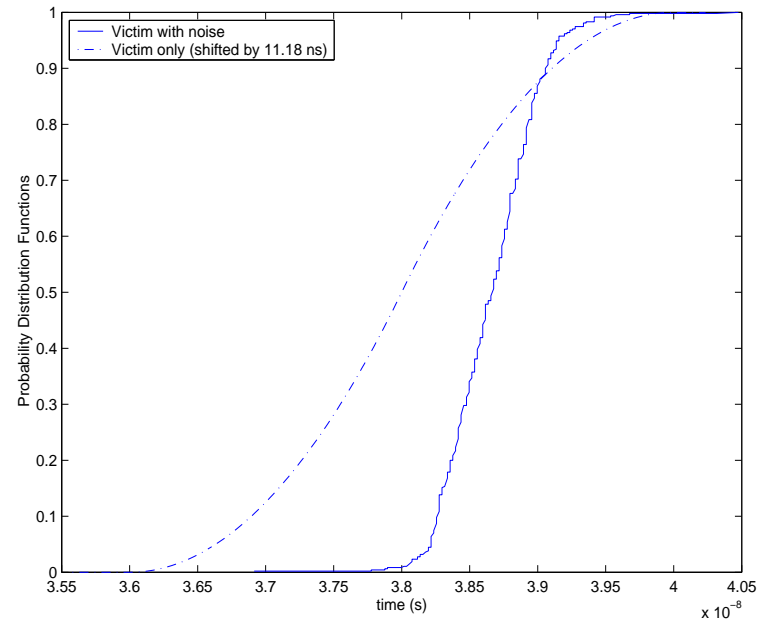

Figure 5: Distributions of the Victim waveform.

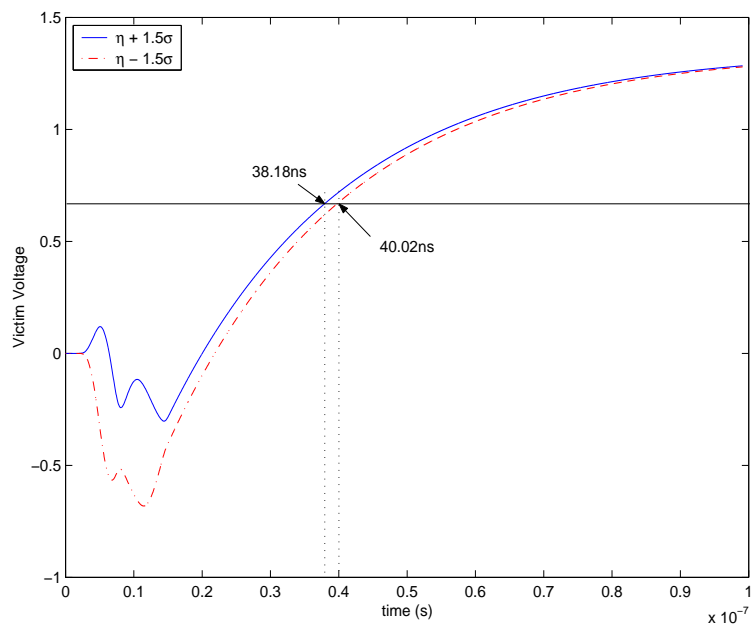

Figure 6: Computation of EAT and LAT

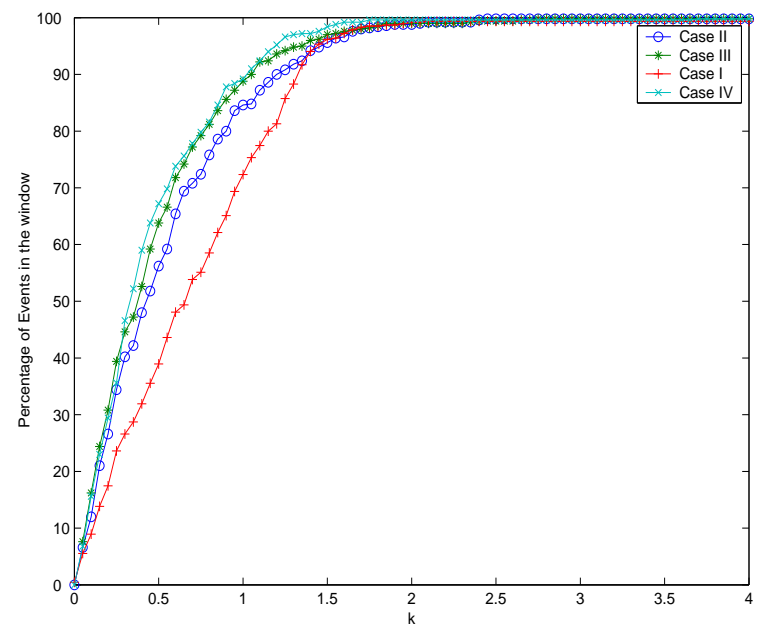

Figure 7: Percentage of Events falling in the window wrt ' $k$ '
[6] P. D. Gross, R. Arunachalam, K. Rajagopal, and L. T. Pileggi. Determination of worst-case aggressor alignment for delay calculation. In Proc. ICCAD, pages 212-219, 1998.

[7] G. Hachtel, R. Jacoby, P. Moceyunas, and C. Morrison. Performance enhancements in bold using 'implications'. In Proc. ICCAD, pages 94-97, 1988.

[8] A. B. Kahng, S. Muddu, and D. Vidhani. Noise and delay uncertainty studies for coupled rc interconnects. In Proc. ASIC/SOC Conf., pages 3-8, 1999.

[9] H. Kawaguchi and T. Sakurai. Delay and noise formulas for capacitively coupled distributed rc lines. In Proc. ASP-DAC, pages 35-43, 1998.

[10] D. Kirkpatrick and A. Sangiovanni-Vincentelli. Digital sensitivity: predicting signal interaction using functional analysis. In Proc. ICCAD, pages 536-541, 1996.

[11] B. Krauter, S. Mehrotra, and V. Chandramouli. Including inductive effects in interconnect timing analysis. In Proc. CICC, pages 445-452, 1999.

[12] W. Kunz and P. R. Menon. Multi-level logic optimization by implication analysis. In Proc. ICCAD, pages 6-13, 1994.

[13] R. Levy, D.Blaauw, G. Braca, A.Dasgupta, A. Grinshpon, O. Chanhee, B. Orshav, S. Sirichotiyakul, and V. Zolotov. ClariNet: A noise analysis tool for deep submicron design. In Proc. DAC, pages 233-238, 2000.

[14] A. Odabasioglu, M. Celik, and L. T. Pileggi. PRIMA : Passive reduced-order interconnect macromodeling algorithm. IEEE Trans. on CAD of Integrated Circuits and Systems, 17(8):645-654, Aug 1998.

[15] L. T. Pillage and R. A. Rohrer. Asymptotic waveform evaluation for timing analysis. IEEE Trans.on CAD of Integrated Circuits and Systems, 9(4):352-366, April 1990.

[16] T. Sakurai. Closed-form expressions for interconnection delay, coupling, and crosstalk in vlsis. IEEE Trans. on Electron Devices, 40(1):118-124, Jan 1993.

[17] S. S. Sapatnekar. On the chicken-and-egg problem of determining the effect of crosstalk on delay in integrated circuits. In Proc. EPEP Conf., pages 245-248, 1999.

[18] Y. Sasaki and G. D. Micheli. Crosstalk delay analysis using relative window method. In Proc. ASIC/SOC Conf., pages 9-13, 1999.

[19] T. Sato, Y. Cao, D. Sylvester, and C. Hu. Characterization of interconnect coupling noise using in-situ delay change curve measurements. In Proc. ASIC/SOC Conf., pages 321-325, 2000.

[20] K. L. Sheppard, V. Narayanan, P. C. Elemendorf, and G. Zheng. Global harmony: Coupled noise analysis for full-chip rc interconnect networks. In Proc. ICCAD, pages 139-146, 1997.

[21] S. Sirichotiyakul, D. Blaauw, O. Chanhee, R. Levy, V. Zolotov, and J. Zuo. Driver modeling and alignment for worst-case delay noise. In Proc. TAU, pages 720-725, 2000.

[22] D. Sylvester and K. Keutzer. Getting to the bottom of deep submicron. In Proc. ICCAD, pages 202-211, 1998.

[23] S. Vrudhula, D. Blaauw, and S. Sirichotiyakul. Estimation of the likelihood of capacitive coupling noise. In Proc. DAC, pages 653-658, 2002.

[24] H. Zhou, N. Shenoy, and W. Nicholls. Timing analysis with crosstalk as fixpoints on complete lattice. In Proc. DAC, pages 714-719, 2001. 